$01 ; 05$

\title{
Температурная зависимость автоволнового механизма пластического течения
}

\author{
(С Л.Б. Зуев, С.В. Колосов, С.А. Баранникова, А.М. Никонова \\ Институт фризики прочности и материаловедения СО РАН, \\ Томск, Россия \\ E-mail: Ibz@ispms.tsc.ru
}

Поступило в Редакцию 24 июля 2020 г.

В окончательной редакции 16 сентября 2020 г.

Принято к публикации 16 сентября 2020 г.

Рассмотрено поведение характеристик автоволн локализованного пластического течения в сплаве $\mathrm{Fe}-\mathrm{Cr}-\mathrm{Ni}$ в интервале температур $143 \leq T \leq 420 \mathrm{~K}\left(0.34 \leq T / \Theta_{\mathrm{D}} \leq 1\right)$, где $\Theta_{\mathrm{D}}=420 \mathrm{~K}-$ температура (параметр) Дебая. Показано, что произведение длины автоволны на скорость ее распространения $\lambda V_{a w}$ в указанном интервале падает с температурой. В то же время отношение этой величины к вязкости фононного газа в металле в исследованном интервале температур остается практически постоянным. Это указывает на независимость упругопластического инварианта деформации от температуры.

Ключевые слова: пластическая деформация, локализация, металлы, температура Дебая.

DOI: $10.21883 /$ PJTF.2020.24.50428.18483

Экспериментальные исследования кинетики развития пластической деформации твердых тел [1] позволили обнаружить главную закономерность этого явления. Она состоит в формировании в ходе процесса автоволн локализованного пластического течения, закономерно эволюционирующих в соответствии с изменениями закона деформационного упрочнения. При этом автоволновые характеристики (длина автоволны локализованной пластической деформации $\lambda$ и скорость ее распространения $\left.V_{a w \mid}\right)$ связаны с решеточными характеристиками деформируемой среды (межплоскостное расстояние, отвечающее максимальной интенсивности рентгеновского рефлекса, $\chi$ и скорость поперечных упругих волн $V_{t}$ ) соотношением, которое для стадии линейного деформационного упрочнения имеет вид

$$
\frac{\lambda V_{a w}}{\chi V_{t}}=\hat{Z} \approx \frac{1}{2}
$$

Природа этого соотношения, названного упругопластическим инвариантом деформации [1], определяется взаимообусловленностью процессов, одновременно протекающих в фононной $\left(\chi\right.$ и $\left.V_{t}\right)$ и деформационной $\left(\lambda\right.$ и $\left.V_{a w}\right)$ подсистемах пластически деформируемой среды. Выполнимость инварианта (1) была установлена на основании опытов по деформации девятнадцати различных металлов, ряда щелочно-галоидных кристаллов и горных пород, а также в экспериментах по ползучести и по измерениям подвижности индивидуальных дислокаций в монокристаллах [1].

Инвариант (1) связывает два пространственновременны́х процесса в деформируемой среде: перераспределение упругих деформаций, характеризуемое распространением ультразвуковых волн, и перераспределение локализованной пластической деформации, описы- ваемое эволюцией автоволн локализованной пластичности. Он играет важную роль в механике пластической деформации, причем многочисленные следствия из него [1] описывают наиболее важные известные закономерности пластической деформации материалов.

На данный момент справедливость соотношения (1) проверена только для условий деформации материалов при $300 \mathrm{~K}$. Поэтому в настоящей работе проведен анализ температурного хода отношения $\lambda V_{a w} / \chi V_{t}$. В экспериментах были использованы вырезанные из листов толщиной $2 \mathrm{~mm}$ плоские образцы поликристаллического аустенитного сплава $\mathrm{Fe}-18$ wt.\% $\mathrm{Cr}-10$ wt.\% Ni с размером рабочей части $40 \times 5 \mathrm{~mm}$ и размером зерна $\sim 12.5 \mu \mathrm{m}$. Образцы растягивались на испытательной машине „Instron-1185“ со скоростью $3.3 \cdot 10^{-4} \mathrm{~s}^{-1}$ при температурах $T=420,296,266,243$, $213,143 \mathrm{~K} \quad\left(T / \Theta_{\mathrm{D}}=1,0.7,0.6,0.57,0.5,0.34\right)$. Здесь $\Theta_{\mathrm{D}} \approx 420 \mathrm{~K}$ - дебаевский параметр (температура Дебая) для железа [2]. Во время испытания образцы находились в прозрачной колбе, где температура поддерживалась постоянной с помощью непрерывной подачи паров азота из сосуда Дьюара. Температура измерялась с помощью хромель-алюмелевой термопары, спай которой находился внутри колбы в контакте с образцом. Температура испытания варьировалась скоростью подачи паров азота, которая регулировалась нагревательным элементом сопротивления, находящимся внутри сосуда Дьюара.

Записанные в ходе выполнения исследования индикаторные кривые в координатах напряжение $\sigma$-деформация $\varepsilon$ преобразовывались в зависимости $s(e)(s-$ истинное напряжение, $e-$ истинная деформация) [3]. Это позволяло надежно выявлять линейные стадии деформационного упрочнения, для которых коэффици- 
Механические характеристики исследованного сплава

\begin{tabular}{c|c|c|c}
\hline$T, \mathrm{~K}$ & $\sigma_{0.2}, \mathrm{MPa}$ & $\varepsilon_{\text {tot }}$ & $\theta \cdot 10^{3}$ \\
\hline 420 & 194 & 0.40 & 8 \\
296 & 269 & 0.70 & 9 \\
266 & 292 & 0.55 & 10.9 \\
243 & 287 & 0.44 & 21.5 \\
213 & 293 & 0.43 & 26.2 \\
143 & 291 & 0.37 & 38.8
\end{tabular}

ент деформационного упрочнения $\theta=E^{-1} d s / d e=\mathrm{const}$ ( $E-$ модуль Юнга) и $s=\theta e$. Значения коэффициентов деформационного упрочнения, а также условного предела текучести $\sigma_{0.2}$ и предельной деформации до разрыва $\varepsilon_{t o t}$ для всех температур, определенные по трем образцам для каждой температуры с точностью 10\%, приведены в таблице.

Непосредственно в ходе механического испытания фотографическим методом регистрировались поля векторов смещений точек поверхности образца $\mathbf{r}(x, y)$ (метод двухэкспозиционной спекл-фотографии [4]). Затем путем численного дифференцирования этих полей по координатам $x$ и $y$ вычислялись продольные, поперечные, сдвиговые и поворотные компоненты тензора пластической дисторсии для всех точек наблюдаемой плоской поверхности образца и строились их пространственные распределения для разных моментов времени [1]. Анализировались распределения продольной компоненты $\varepsilon_{x x}$ тензора пластической дисторсии. Это позволяло измерить характеристики автоволны локализованной пластичности $\lambda$ и $V_{a w}$, которые были постоянными на исследуемых стадиях линейного деформационного упрочнения.

Температурная зависимость произведения $\lambda V_{a w}$ показана на рис. 1,a. Анализ экспериментальных данных показал, что длина автоволны в пределах точности эксперимента от температуры не зависит и составляет $\lambda=4.5 \pm 0.3 \mathrm{~mm}$. Таким образом, зависимости $\lambda V_{a w}(T)$, приведенные на рис. 1, эквивалентны зависимостям $V_{a w}(T)$. На рис. $1, b$ показано, что для области $1<\Theta_{\mathrm{D}} / T<2$ зависимость $V_{a w}(T)$ удовлетворительно спрямляется в координатах $\ln \left(\lambda V_{a w}\right)-T^{-1}$.

Полученные данные о скоростях движения фронтов локализованной пластичности прежде всего позволили проверить форму связи коэффициента деформационного упрочнения (см. таблицу) и скорости распространения автоволн локализованной пластичности, установленную ранее [1] и имеющую вид

$$
V_{a w}=V_{0}+\frac{\Xi}{\theta} \sim \theta^{-1} \text {. }
$$

Как показывают данные рис. 2, указанное соотношение выполняется в исследованном интервале температур, а определенные в этом случае значения входящих в уравнение (2) констант $V_{0}=1.8 \cdot 10^{-5} \mathrm{~m} / \mathrm{s}$ и $\Xi=9.8 \cdot 10^{-7} \mathrm{~m} / \mathrm{s}$ близки к найденным ранее значениям,
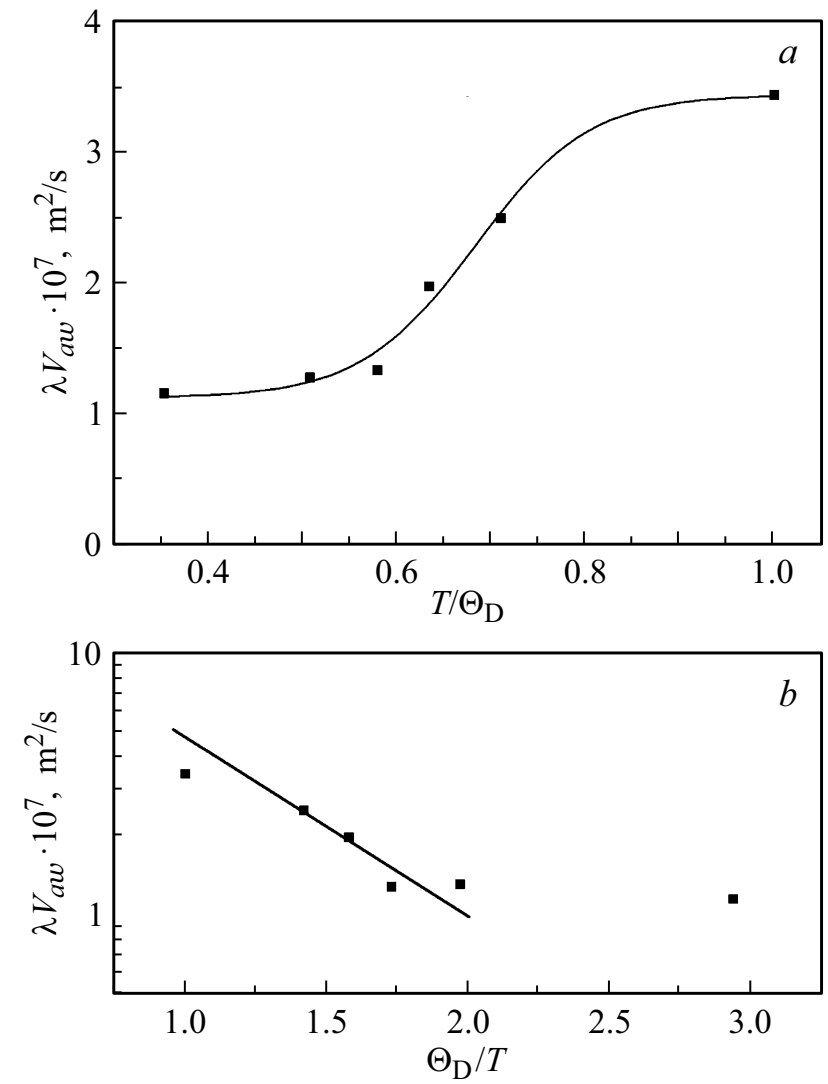

Рис. 1. Температурная зависимость величины $\lambda V_{a w}(a)$ и та же зависимость в координатах $\ln \left(\lambda V_{a w}\right)-T^{-1}(b)$.

общим для всех исследованных ранее материалов, полученным при $T=300 \mathrm{~K}[1]$.

Из рис. $1, b$ следует, что температурная зависимость $V_{a w}$ (как и $\left.\lambda V_{a w}\right)$ удовлетворительно аппроксимируется соотношением Аррениуса для термически активированных процессов

$$
V_{a w} \sim\left(\lambda V_{a w}\right) \sim \exp \left(-\frac{H}{k_{\mathrm{B}} T}\right),
$$

где $H=H(\sigma) \approx U_{0}-\gamma \sigma-$ энтальпия активации процесса, $k_{\mathrm{B}}$ - постоянная Больцмана, $U_{0}$ - высота потенциального барьера, а $\gamma$ - активационный объем [5]. Аппроксимация, показанная на рис. $3, a$, приводит к $U_{0} \approx 0.06 \mathrm{eV}$, а $\gamma \approx 8.5 b^{3}$, где $b-$ вектор Бюргерса дислокаций в ГЦК-железе. Низкие значения активационных параметров процесса означают, что автоволны локализованной пластичности могут генерироваться в деформируемых кристаллах при любом режиме деформирования, как это и наблюдается в действительности [1].

Анализируя зависимость $\lambda V_{a w}(T)$, показанную на рис. 1, заметим, что произведение $\lambda V_{a w}$ в уравнении (1) имеет размерность коэффициента переноса $\left(\mathrm{m}^{2} / \mathrm{s}\right)$, в частности кинематической вязкости среды. Тогда соотношение (1) приобретает смысл аналога числа Рейнольдса [7], характеризующего режимы ламинарного и 


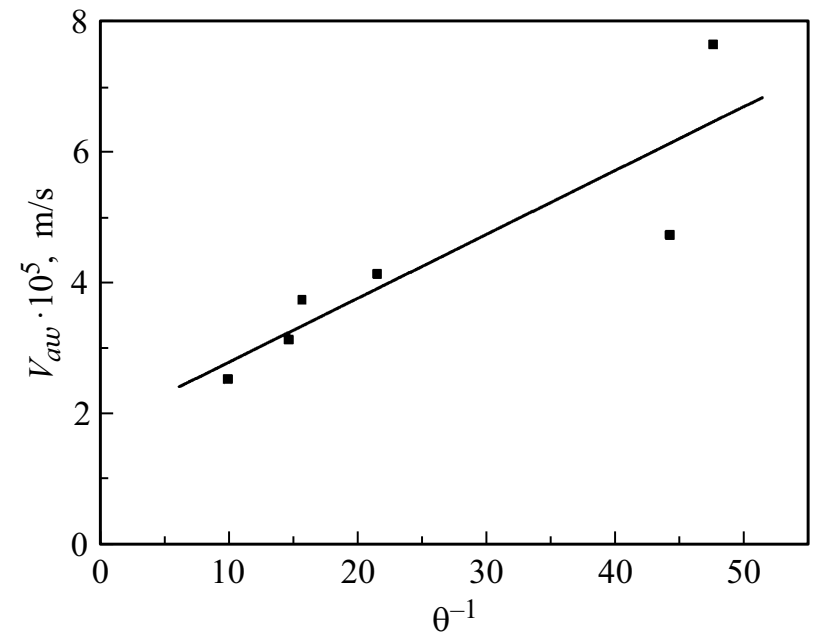

Рис. 2. Скорость распространения автоволн локализованной пластичности при разных температурах как функция коэффициента деформационного упрочнения.

турбулентного движения жидкости и записываемого в виде

$$
\operatorname{Re}=\frac{l V}{v},
$$

где $l$ - характерный пространственный масштаб потока, $V$ - его скорость, а $v$-кинематическая вязкость среды. В случае пластического течения можно полагать, что $l \equiv \lambda, V \equiv V_{a w}$ и $v \equiv \chi V_{t}$. Идея о возможности реализации ламинарного и турбулентного течения при пластической деформации по аналогии с течением жидкостей была высказана еще Коттреллом [8], но без количественного обоснования. В случае пластического течения условие $\hat{Z} \approx 1 / 2$ отвечает пластической деформации с линейным законом деформационного упрочнения.

Далее рассмотрим температурный ход величин $\lambda V_{a w}$ и кинематической вязкости $\chi V_{t}$, входящих в уравнение $(1)$. При этом будем полагать, что в основе автоволновых механизмов пластического течения, как и в основе всех механизмов пластичности вообще [9], лежит движение дислокаций. Известно, что скорость движения последних под действием приложенного напряжения контролируется вязкостью фононного газа $[6,10]$ (в металлах также и электронного [11]) в соответствии с соотношением $V_{d i s l}=(b / B) \sigma$, где $B-$ константа торможения дислокаций.

Число Рейнольдса может быть записано также в форме [7]:

$$
\operatorname{Re}=\frac{\rho l V}{\mu}
$$

где $\rho-$ плотность жидкости, а $\mu-$ ее динамическая вязкость. При ,деформационной“ интерпретации соотношения (5) можно полагать, что $\chi V_{t} \sim \mu \sim B$, и на этом основании отождествить величину $\mu$ с вязкостью $B$ фононного газа, контролирующего подвижность дислокаций при их надбарьерном (квазивязком) движении [7]. Это утверждение немедленно допускает возможность обсуждать температурную зависимость числа Рейнольдса и соответственно инварианта $\hat{Z}$ на основе температурной зависимости $B(T)$, приведенной и объясненной в работе [6].

Поэтому сопоставим показанные на рис. $3, b$ экспериментально полученную зависимость $\lambda V_{a w}(T)$ (кривая 1$)$ и зависимость $\frac{B_{T}}{B_{T=\Theta_{\mathrm{D}}}}(T)$ (кривая 2). Исходные данные для построения последней зависимости взяты из работы [6]. В приведенном соотношении $B_{T}-$ константа торможения дислокаций при температуре $T$, а $B_{T=\Theta_{\mathrm{D}}}-$ то же при $T=\Theta_{\mathrm{D}}$. Как показывают экспериментальные и литературные данные, при падении температуры испытания от $T / \Theta_{\mathrm{D}}=1$ до 0.3 значения зависимостей $\lambda V_{a w}(T)$ и $\frac{B_{T}}{B_{T=\Theta_{\mathrm{D}}}}(T)$ также уменьшаются в одинаковой степени, т.е. приближенно выполняется соотношение $\lambda V_{a w} / \chi V_{t} \approx$ const. Это постоянство можно рассматривать как указание на температурную независимость упругопласического инварианта (1).

Кроме того, определенные перспективы возникают в связи с представлением упругопластического инварианта деформации как аналога числа Рейнольдса. Такой подход может оказаться перспективным и полезным для
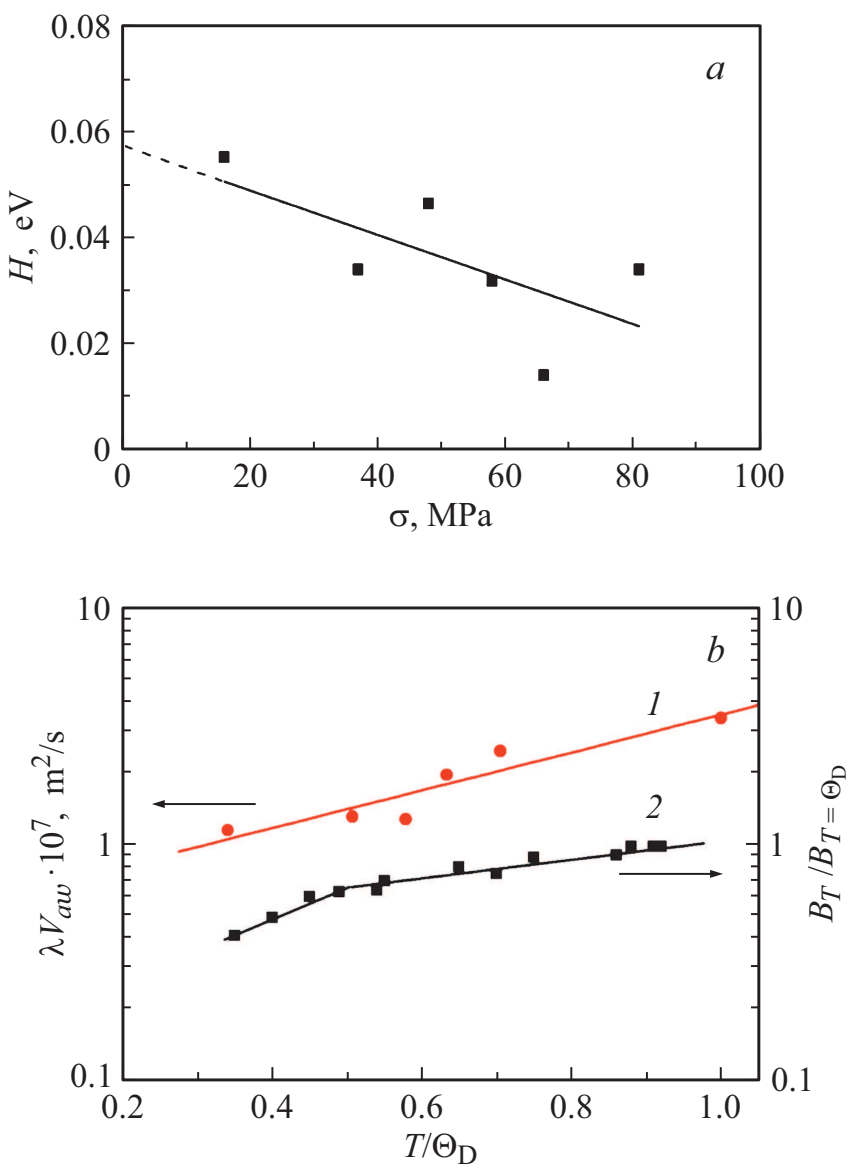

Рис. 3. Определение термоактивационных параметров развития автоволн локализованной пластичности $(a)$ и сопоставление зависимости $\lambda V_{a w}(T)(1)$ и зависимости $\frac{B_{T}}{B_{T=\Theta_{\mathrm{D}}}}(T)$ (2), построенной по данным [6] $(b)$. 
выяснения физической природы развития локализованного пластического течения в материалах.

\section{Финансирование работы}

Работа выполнена в рамках государственного задания ИФПМ СО РАН (проект III.23.1.2).

\section{Конфликт интересов}

Авторы заявляют, что у них нет конфликта интересов.

\section{Список литературы}

[1] Zuev L.B., Barannikova S.A. // Crystals. 2019. V. 9. P. 458488. DOI: $10.3390 /$ cryst 9090458

[2] Newnham R.E. Properties of materials. Oxford: University Press, 2005. $378 \mathrm{p}$.

[3] Asaro R.J., Lubarda V.A. Mechanics of solids and materials. Cambridge: Cambridge University Press, 2006. 860 p. DOI: 10.1016/j.matchar.2007.01.010

[4] Asundi A. // Handbook of optical metrology: principles and applications / Ed. T. Yoshizawa. Boca Raton: CRC Press, 2015. P. 583-603. DOI: $10.1201 / 9781420019513$

[5] Caillard D., Martin J.L. Thermally activated mechanisms in crystal plasticity. Oxford: Elsevier, 2003. 433 p.

[6] Al'shits V.I., Indenbom V.L. // Dislocations in crystals. V. 7 / Ed. F.R.N. Nabarro. Amsterdam: North-Holland, 1986. P. 43111.

[7] Климонтович Ю.Л. Введение в физику открытых систем, М.: Янус-К, 2002. 284 с.

[8] Cottrell A.H. Dislocations and plastic flow in crystals. Oxford: Clarendon Press, 1953. 223 p. [Коттрелл A.X. Дислокации и пластическое течение в кристаллах. М.: Металлургия, 1958. 267 c.].

[9] Landau P., Makov G., Shneck R.Z., Venkert A. // Acta Mater. 2011. V. 59. P. 5342-5350.

DOI: 10.1016/j.actamat.2011.04.064

[10] Blaschke D., Motolla E., Preston D.L. // Phil. Mag. 2020. V. 100. P. 571-600. DOI: 10.1080/14786435.2019.1696484

[11] Ziman J.M. Electrons and phonons. Oxford: University Press, 2001. 554 p. 